\section{Lungenemphysem: Positive Effekte durch Lungenresektion?}

van Geffen WH et al. Surgical and endoscopic interventions that reduce lung volume for emphysema: a systemic review and meta-analysis. Lancet Respir Med 2019; doi:10.1016/S22132600(18)30431-4

Patienten mit schwerem Lungenemphysem haben eine stark begrenzte Anzahl an Therapieoptionen; die chirurgische oder endoskopische Volumenresektion stellt eine von ihnen dar. Obgleich einige Patienten von diesem Eingriff zu profitieren scheinen, sind die Effekte auf das klinische Behandlungsergebnis bis heute unklar. Van Geffen und Team wollten hier eine Lücke schließen, und präsentieren nun eine systematische Übersichtsarbeit samt Metaanalyse.

Die chronisch obstruktive Lungenerkrankung (COPD) gehört weltweit zu den häufigsten pulmonalen Erkrankungen, viele betroffene Patienten entwickeln im Verlauf ein Lungen Emphysem. Da insbesondere die konservativen Behandlungsoptionen stark begrenzt sind und Patienten im Krankheitsverlauf massiv in ihrer Lebensqualität eingeschränkt werden, bietet sich in bestimmten Fällen eine chirurgische oder auch endoskopische Volumenresektion an. Da Vor- und Nachteile dieser invasiven Eingriffe bis heute diskutiert werden, haben van Geffen und sein Team nun die wichtigsten randomisierten und kontrollierten klinischen Studien der letzten Jahre zum Thema im Rahmen einer systemischen Übersichtsarbeit und Metaanalyse zusammengefasst.

Sie führten dabei zunächst eine Literaturrecherche in den Datenbanken PubMed und Embase durch und stellten für potenziell geeignete Studien die folgenden Einschlusskriterien auf:

- Randomisierte kontrollierte Studien,

- Publikationssprache Englisch,

- Vergleich einer Intervention zur

Volumenreduktion mit einer Kontrollgruppe.
Die Studienpatienten sollten

- älter als 35 Jahre sein,

- eine post-bronchodilatatorische FEV 1 von unter $60 \%$ des prädiktiven Wertes haben,

- sowie ein Residualvolumen von über $150 \%$.

Für die Metaanalyse extrahierten die Forscher alle Daten von Patienten, die die obigen Kriterien erfüllten und verglichen schließlich die gepoolte Interventionsgruppe mit den Kontrollpatienten. Als primären klinischen Endpunkt definierten sie das Residualvolumen, die $\mathrm{FEV}_{1}$, das Ergebnis des St. George's Respiratory Questionnaire sowie das Resultat des 6Minuten-Gehtestes.

Sekundäre Endpunkte waren u. a. schwere Nebenwirkungen einschließlich Mortalität, Sterblichkeit innerhalb der ersten 45 Tage nach dem Eingriff, sowie die Gesamtmortalität. Bias-Risiko allgemein und Publikationsbias beurteilten die Autoren mit Hilfe des Review Managers Version 5,3. Darüber hinaus kam das Cochrane „Risk of Bias Tool“ für randomisierte kontrollierte Studien zum Einsatz.

\section{Eingriff verbesserte Lungenfunktion}

Die systematische Literaturrecherche ergab 4747 Treffer, die Daten von 2794 Patienten aus 20 Studien gingen schließlich in die quantitative Analyse mit ein. Die Forscher fanden keinen Anhalt für signifikante Publikationsbias, das Bias-Risiko musste allerdings für einzelne Parameter als hoch eingestuft werden und wurde von den Forschern als Limitation der Analyse bewertet. In 7 Studien erhielten die Patienten eine chirurgische Volumenreduktion, in 6 Studien kam ein endobronchiales Verfahren zum Einsatz. In Hinblick auf die einzelnen detaillierten Methoden mussten van Geffen und Kollegen eine große Heterogenität der Untersuchungen feststellen.

Durch eine Intervention reduzierte sich das Residualvolumen im Vergleich zur Kontrolle um durchschnittlich $0,58 \mathrm{Li}$ ter $(95 \%$ Konfidenzintervall von $-0,8$ bis $-0,37)$. Weiterhin stieg die $\mathrm{FEV}_{1}$ auf
$15,87 \%(12,27$ bis $19,47 \%)$ an, und die Leistung im 6-Minuten-Gehtest steigerte sich im Mittel um 43,28m (31,36 bis $55,21)$. Auch für die Lebensqualität (St. George's Respiratory Questionnaire) konnten die Autoren positive Effekte einer Volumenresektion konstatieren.

Die Odds Ratio für eine schwere Komplikation oder Nebenwirkung einschließlich Versterben durch die Intervention musste auf 6,21 (4,02 bis 9,58) beziffert werden. Aufgrund der überwiegend signifikant positiven Effekte einer Volumenreduktion bei Patienten mit fortgeschrittenem Lungenemphysem halten die Studienautoren diese Intervention trotz großer Heterogenität der einzelnen Studien und einem nicht unerheblichen Bias-Risiko für eine klinisch relevante Therapieoption.

\section{FAZIT}

In dieser Metaanalyse konnte eine chirurgische oder endoskopische Volumenresektion das Outcome von Patienten mit fortgeschrittenem Lungenemphysem deutlich verbessern. Bei vergleichbarer Mortalitätsrate zeigten Patienten nach einer Intervention u.a. Verbesserungen hinsichtlich $\mathrm{FEV}_{1}$, Symptomschwere und Lebensqualität. Die Autorinnen/Autoren weisen allerdings auf eine hohes Bias-Risiko und starke Heterogenität der Studien hin und empfehlen daher weitere prospektive Untersuchungen.

Dipl.-Psych. Annika Simon, Hannover 